\title{
microRNA expression profiles in oral squamous cell carcinoma
}

\author{
DAISUKE SOGA, SAYAKA YOSHIBA, SUNAO SHIOGAMA, HIROAKI MIYAZAKI, \\ SEIJI KONDO and SATORU SHINTANI
}

\begin{abstract}
Department of Oral and Maxillofacial Surgery, Showa University School of Dentistry, Ota-ku, Tokyo 145-8515, Japan
\end{abstract}
Received December 12, 2012; Accepted January 31, 2013

DOI: $10.3892 / o r .2013 .2488$

\begin{abstract}
RNAs (miRNAs) are involved in cancer pathogenesis, apoptosis and cell growth, thereby functioning as both tumor suppressors and oncogenes. However, the expression patterns and roles of miRNAs in oral squamous cell carcinoma (OSCC) remain largely unknown. We hypothesized that oral cancer may have a unique miRNA profile, which in turn may play a critical role in oral cancer development, progression, diagnosis and prognosis. We, therefore, investigated the expression profiles of 29 OSCC tumors and 7 normal oral mucosal samples. The miRNA expression patterns in OSCC were examined by TaqMan-based microRNA assays. We were subsequently able to identify the candidates of cancer-related miRNAs through analysis of the miRNA expression profiles. In conclusion, OSCC tissues were shown to have a unique miRNA profile pattern when compared with that in normal tissues. The present study may provide useful information for further investigation of the functional roles of miRNAs in OSCC development, progression, diagnosis and prognosis.
\end{abstract}

\section{Introduction}

Oral cancer, which is occurring at an increasing frequency worldwide, is the sixth most common malignancy in humans. This year alone, it is estimated that $\sim 600,000$ new cases will arise, with only $40-50 \%$ of patients with oral squamous cell carcinoma (OSCC) likely to survive for 5 years. Despite combined surgery and radiation therapy, long-term survival of patients with oral cancer has shown no improvement over the past few decades $(1,2)$. This is primarily due to the poor clinical prognosis of patients with lymph node metastasis. A deeper understanding of the molecular basis of the highly malignant properties of oral cancer combined with patient stratification is therefore needed.

Correspondence to: Dr Daisuke Soga, Department of Oral and Maxillofacial Surgery, Showa University School of Dentistry, 2-1-1 Kitasenzoku, Ota-ku, Tokyo 145-8515, Japan

E-mail: d-soga@dent.showa-u.ac.jp

Key words: microRNA, exhaustive analysis, abnormal expression, oral squamous cell carcinoma
microRNAs (miRNAs) are non-coding small RNAs ( 22 nucleotides) that regulate post-transcriptional gene expression by interfering with the translation of target mRNAs. A single miRNA can regulate the expression of several genes, while more than one-third of all protein-coding genes are thought to be under translational control of miRNAs. miRNAs are involved in a variety of cellular processes, including the regulation of cellular differentiation, proliferation and apoptosis $(3,4)$. In addition, aberrant expression of miRNAs is known to induce various human malignancies, clearly classified by their miRNA profiles $(5,6)$. However, little is known about the miRNA expression patterns or function in oral cancer $(7,8)$.

Specific overexpression or underexpression of miRNAs has been correlated with particular types of tumors $(5,9,10)$. It has also been suggested that miRNA overexpression could result in downregulation of tumor-suppressor genes, while underexpression may lead to oncogene upregulation (9). Most importantly, it has been suggested that miRNA expression signatures could be used to predict the outcome in several tumor types, including lung cancer and chronic lymphocytic leukemia, as well as to predict the response to chemotherapy. miRNAs may therefore prove to be novel therapeutic targets for a wide range of diseases, including cancer (11).

In the present study, we examined the expression profiles of miRNAs in OSCC, revealing differential expression between normal and cancer tissues. These findings may further facilitate the potential therapeutic and diagnostic use of miRNAs in OSCC.

\section{Materials and methods}

Clinical samples and cell lines. Twenty-nine OSCC samples and 7 oral mucosal samples were obtained from oral cancer patients who underwent surgery at Showa University from April 2004 to November 2008. Written informed consent was obtained from all patients prior to sampling, and samples were maintained at $-80^{\circ} \mathrm{C}$ until use. OSCC samples were microscopically examined for determination of cancer cell content by two independent pathologists; they were dissected to enrich cancer cells when necessary. For molecular analysis, 29 samples containing $\geq 50 \%$ cancer cells were used. Normal oral mucosa was extracted at a sufficient margin from the cancer, and the absence of a muscle layer and cancer cells was confirmed microscopically. The present study was certified by the Ethics Committee of the Showa University. 
RNA preparation. miRNA was prepared with the Recover All Total Nucleic Acid Isolation kit (Applied Biosystems, Darmstadt, Germany) according to the kit protocol. RNA yield and the A260/280 ratio were monitored with a NanoDrop ND-100 spectrometer (NanoDrop Technologies, Wilmington, DE, USA).

Reverse transcription. miRNAs were reverse transcribed with the TaqMan miRNA Reverse Transcription kit (Applied Biosystems), using $60 \mathrm{ng}$ total RNA and pools of miRNA specific stem-loop primers (Megaplex RT Primers Pool A and B; Applied Biosystems). After reverse transcription, the cDNA was preamplified with Megaplex PreAmp Primers (Pool A and B) according to the recommendations of the supplier (Applied Biosystems).

TaqMan low density arrays. Differential expression of 768 miRNAs was determined using Taqman microRNA Array v2.0 (Applied Biosystems), with an A and B card for each. The array plate also included the RNU48 transcript as a normalization signal. Expression levels of each mature miRNA were evaluated using the comparative threshold cycle $(\mathrm{Ct})$ method, with normalization to RNU48 $\left(2^{-\Delta \Delta C t}\right)$. The fold-change in each miRNA was calculated from the difference in expression levels between tumor tissues and normal tissues.

miRNA target prediction. TargetScan (http://www.targetscan. org/) was used to analyze potential target genes of the deregulated miRNAs.

\section{Results}

Differential expression of miRNAs between normal oral mucosa and oral cancer tissues. To define the role of miRNAs in OSCC, we investigated the expression profiles of 29 OSCC tumors and 7 normal oral mucosal samples. The 29 OSCC tissue samples were of various stages and histological grades (Table I). TaqMan-based microRNA assays of 768 different miRNA targets were performed, revealing a total of 177 detectable miRNAs. Volcano plots show differential expression between normal and cancer tissues (Fig. 1). We subsequently calculated the degree of differential expression, revealing that miRNAs $>4$-fold upregulated or downregulated could be considered as candidate miRNAs. miR-31*, miR-31, miR-135b, miR-193a-5p, miR-103, miR-224, miR-93, miR-200c, miR-183, miR203, miR-21 and miR-223 were shown to be upregulated, while miR-133a, miR-376c, miR-411, miR-30a-3p, miR-489, miR-139-5p, miR-483-5p, miR-30e-3p, miR-409-3p, let-7c and miR-486-5p were shown to be downregulated in OSCC (Tables II and III).

Differential expression of miRNAs between non-metastatic (-) and metastatic (+) OSCC. As cervical lymph node metastasis is directly correlated with oral cancer prognosis, we subsequently aimed to determine whether miRNA expression was associated with outcome in OSCC patients. Volcano plots show the differential expression of miRNAs in non-metastatic (-) and metastatic (+) OSCC (Fig. 2). We subsequently calculated the level of significance of the differential expression between these groups. miR-489, miR-483-5p and miR-1291
Table I. Demographic and clinical features of the OSCC patients.

\begin{tabular}{|c|c|}
\hline Characteristic & $\mathrm{n}(\%)$ \\
\hline \multicolumn{2}{|l|}{ Gender } \\
\hline Male & $20(69)$ \\
\hline Female & $9(31)$ \\
\hline \multicolumn{2}{|l|}{ Smoking habit } \\
\hline Positive & $8(28)$ \\
\hline Negative & $21(72)$ \\
\hline \multicolumn{2}{|l|}{ Drinking habit } \\
\hline Positive & $9(31)$ \\
\hline Negative & $20(69)$ \\
\hline \multicolumn{2}{|c|}{ WHO classification } \\
\hline Well & $10(34)$ \\
\hline Moderate & $8(28)$ \\
\hline Poor & $11(38)$ \\
\hline \multicolumn{2}{|l|}{ YK grade } \\
\hline 1 & $1(4)$ \\
\hline 2 & $3(10)$ \\
\hline 3 & $13(45)$ \\
\hline 4 & $12(41)$ \\
\hline \multicolumn{2}{|c|}{ Local recurrence } \\
\hline Positive & $10(34)$ \\
\hline Negative & $19(66)$ \\
\hline \multicolumn{2}{|l|}{ Age (years) } \\
\hline$>65$ & $21(72)$ \\
\hline$\leq 65$ & $8(28)$ \\
\hline \multicolumn{2}{|l|}{ Stage } \\
\hline $\mathrm{I}$ & $6(21)$ \\
\hline II & $9(31)$ \\
\hline III & $3(10)$ \\
\hline IV & $11(38)$ \\
\hline \multicolumn{2}{|l|}{$\mathrm{T}$ status } \\
\hline $\mathrm{I}$ & $6(21)$ \\
\hline II & $14(48)$ \\
\hline III & $2(7)$ \\
\hline IV & $7(24)$ \\
\hline \multicolumn{2}{|l|}{$\mathrm{N}$ status } \\
\hline NO & $15(51)$ \\
\hline $\mathrm{N} 1$ & $4(13)$ \\
\hline $\mathrm{N} 2$ & $10(36)$ \\
\hline
\end{tabular}

were shown to be downregulated in OSCC (Table IV). In addition, a decision tree model was used to classify the two groups (Fig. 3), doing so with $82 \%$ accuracy.

\section{Discussion}

miRNAs are a new class of non-coding small RNAs that regulate cell proliferation and various cellular functions by interfering with the translation of the target mRNAs $(3,4,12,13)$. Recent studies have demonstrated that altered expression of miRNAs induces various human malignancies $(14,15)$. 


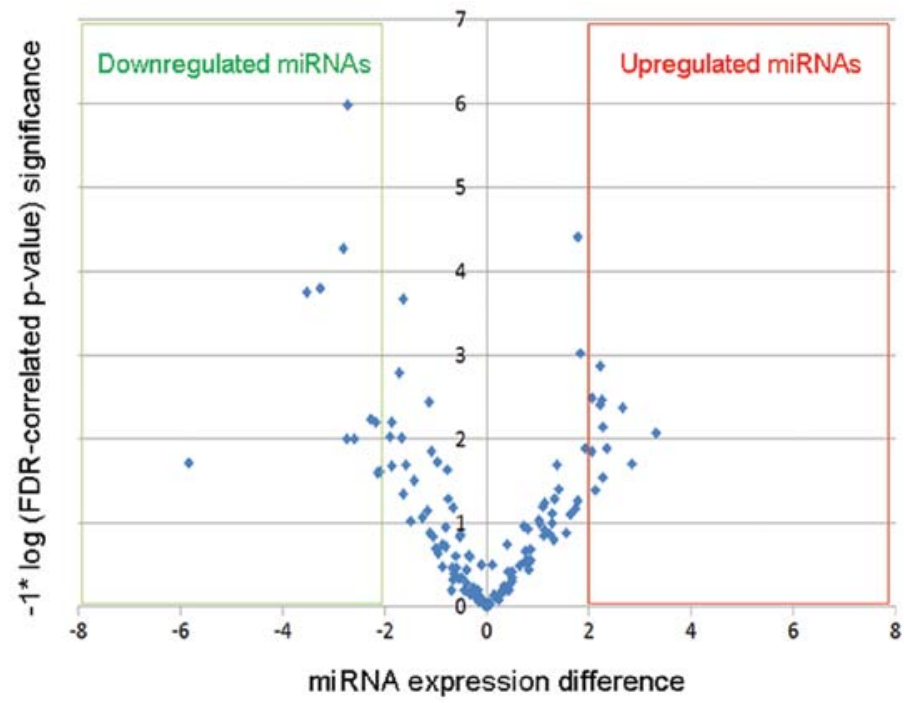

Figure 1. Comparison of miRNA expression between OSCC and normal oral mucosa. Volcano plots of 177 miRNAs analyzed for comparison between OSCC and normal oral mucosa. The level of differential miRNA expression between OSCC and normal oral mucosa is plotted on the x-axis, and the P-value of a FDR-corrected Wilcoxon signed-rank test of the differences $(-1 * \log 10$ scale) is indicated on the y-axis. miRNAs significantly different between the two groups are boxed in red and green.

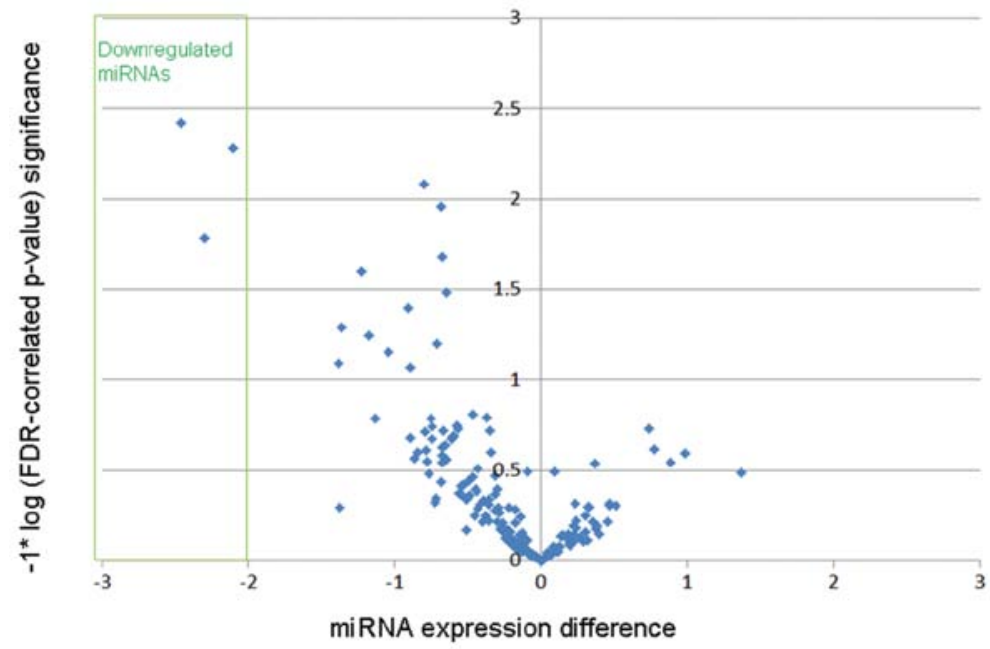

Figure 2. Comparison of miRNA expression between non-metastatic and metastatic OSCC. Volcano plots of 177 miRNAs analyzed for comparison between non-metastatic and metastatic OSCC. The level of differential miRNA expression between non-metastatic and metastatic OSCC is plotted on the x-axis, and the P-value of a FDR-corrected Wilcoxon signed-rank test of the differences $(-1 * \log 10$ scale) is indicated on the y-axis. miRNAs significantly different between the two groups are boxed in green.

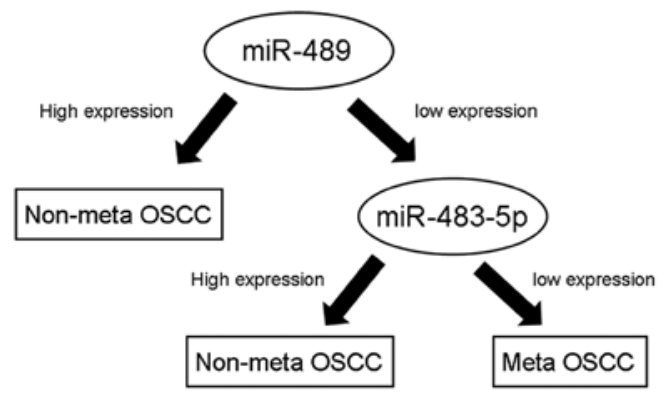

Figure 3. A decision making model was constructed to classify the oral cancer samples according to metastasis using miRNA expression using a training set of oral cancers $(\mathrm{n}=29)$. miR-489 (Ct-value $\leq 10.8$; high expression) and miR-483-5p (Ct-value $\leq 12.1$; high expression) resulted in the most accurate prediction ( $82 \%$ accuracy) for classifying the samples into the two corresponding groups.
However, little is known about the role of miRNAs in OSCC. In this study, we investigated the miRNA expression profiles of normal oral mucosa and OSCC tissues. miRNA profiling in B cell lymphoma (16), prostate (17), and colon cancer (18) was previously performed; however, to the best of our knowledge, this is the first comprehensive miRNA profiling study of oral cancer through exhaustive analysis.

miRNA analysis was performed using TaqMan microRNA Array v2.0, which consists of 768 candidate miRNA sites. A total of 177 miRNAs were detected in the oral cancer tissues and normal oral mucosa. Volcano plot analysis subsequently revealed aberrant expression of miRNAs in OSCC (Fig. 2). This result suggests that miRNA expression profiles differ between normal and abnormal cells. 
Table II. Differentially expressed miRNAs showing increased expression in OSCC compared to normal mucosa.

\begin{tabular}{lcccl}
\hline microRNA & Fold-change $(\mathrm{Ct}-\mathrm{Ca} / \mathrm{Ct}-\mathrm{N})$ & FDR & Chromosome location & \\
\hline hsa-miR-31* & 0.60 & 0.008 & 9p21.3 & RSBN1, ARHGEF2, IDE, NR5A2, SH2D1A \\
hsa-miR-31 & 0.29 & 0.020 & $9 \mathrm{p} 21.3$ & RSBN1, ARHGEF2, IDE, NR5A2, SH2D1A \\
hsa-miR-135b & 0.68 & 0.004 & $1 \mathrm{q} 32.1$ & ANGPT2, GK5, NR3C2, GULP1, LOC221710 \\
hsa-miR-193a-5p & 0.77 & 0.013 & $17 \mathrm{q} 11.2$ & HSPB6, ZNF385C, ITSN1, OLIG3, USO1 \\
hsa-miR-103 & 0.76 & 0.007 & $5 q 34$ & DICER1, TMEM16C, NF1, FOXP1, HRB \\
hsa-miR-224 & 0.71 & 0.029 & Xq28 & ZDHHC20, AFF3, U2SURP, C80r444, TTC3 \\
hsa-miR-93 & 0.64 & 0.003 & $7 \mathrm{q} 22.1$ & FGD4, PKD2, MAP3K2, ZNFX1, PDCD1LG \\
hsa-miR-200c & 0.27 & 0.004 & $12 \mathrm{p} 13.31$ & ZEB1, FAM122C, ZEB2, LRP1B, WIPF1 \\
hsa-miR-183 & 0.82 & 0.001 & $7 \mathrm{q} 32.2$ & ABAT, AKAP12, PIGX, PTPN4, REV1 \\
hsa-miR-203 & 0.22 & 0.040 & $14 q 32.33$ & ZNF281, CAMTA1, B3GNT5, LIFR, ABCE1 \\
hsa-miR-21 & 0.54 & 0.014 & $17 q 23.1$ & ANF367, GPR64, YOD1, PHF14, PLEKHA1 \\
hsa-miR-223 & 0.27 & 0.003 & Xq12 & FBXW7, SP3, PAX6, C13orf31, PURB
\end{tabular}

Fold-changes are expressed as the ratio of OSCC Ct values vs. normal mucosa $\mathrm{Ct}$ values. FDR, false discovery rate. The top putative targets identified with TargetScan are included.

Table III. Differentially expressed miRNAs showing decreased expression in OSCC compared to normal mucosa.

\begin{tabular}{lcccl}
\hline microRNA & Fold-change (Ct-Ca/Ct-N) & FDR & Chromosome location & \multicolumn{1}{c}{ Putative target } \\
\hline hsa-miR-133a & 3.13 & 0.019 & $18 \mathrm{q} .11 .2$ & SYT2, LHFP, CCBL2, BRUNOL4, TTPAL \\
hsa-miR-376c & 1.55 & $<1 \mathrm{e}-03$ & $14 \mathrm{q} 32.31$ & ARFGEF1, PAPSS2, GABRG2, SYF2, ARFGEF2 \\
hsa-miR-411 & 1.52 & $<1 \mathrm{e}-03$ & $14 \mathrm{q} 32.31$ & ELFN1, SLC4A7, C16orf52, C21orf91, SPRY4 \\
hsa-miR-30a-3p & 1.75 & $<1 \mathrm{e}-03$ & $6 \mathrm{q} 13$ & NUFIP2, ZNF85, RUNDC2B, FIGN, POU4F1 \\
hsa-miR-489 & 1.36 & 0.010 & $7 \mathrm{q} 21.3$ & ETNK1, PARM1, CWC25, NRIP1, PNISR \\
hsa-miR-139-5p & 1.65 & $<1 \mathrm{e}-03$ & $11 \mathrm{q} 13.4$ & TMF1, USP6NL, TBX1, SCAPER, NDRG2 \\
hsa-miR-483-5p & 1.33 & 0.010 & $11 \mathrm{p} 15.5$ & SELO, MPZ, SRSF4, SLC12A5, RNF165 \\
hsa-miR-30e-3p & 1.54 & 0.006 & $1 \mathrm{p} 34.2$ & NUFIP2, ZNF85, RUNDC2B, POU4F1, DSN \\
hsa-miR-409-3p & 1.42 & 0.006 & $14 \mathrm{q} 32.31$ & MRPL35, LRRN4CL, POMP, MTF2, TMEM65 \\
hsa-let-7c & 1.29 & 0.025 & $21 \mathrm{q} 21.1$ & C14orf28, FIGNL2, HMGA2, LIN28B, TRIM71 \\
hsa-miR-486-5p & 1.49 & 0.025 & $8 \mathrm{p} 11.21$ & FOXO1, GPX8, PTEN, TRAPPC6B, TWF1 \\
\hline
\end{tabular}

Fold-changes are expressed as the ratio of OSCC Ct values vs. normal mucosa Ct values. FDR, false discovery rate. The top putative targets identified with TargetScan are included.

Table IV. Differentially expressed miRNAs showing decreased expression in non-metastatic and metastatic OSCC.

\begin{tabular}{lcccc}
\hline microRNA & Fold-change (Ct-Ca/Ct-N) & FDR & Chromosome location & Putative target \\
\hline hsa-miR-489 & 1.27 & 0.004 & $7 \mathrm{q} 21.3$ & ETNK1, ALS2CR13, HRH4, LONRF2, SFRS7 \\
hsa-miR-1291 & 1.18 & 0.017 & $12 \mathrm{q} 13.11$ & AQP1, ARID3B, MECP2, MAP3K9, PGM5 \\
hsa-miR-483-5p & 1.22 & 0.005 & $11 \mathrm{p} 15.5$ & RAB3IP, ZBTB26, PGAM1, C5orf42, RBAK \\
\hline
\end{tabular}

Fold-changes are expressed as the ratio of OSCC Ct values vs. normal mucosa Ct values. FDR, false discovery rate. The top putative targets identified with TargetScan are included.

To identify candidate cancer-related miRNAs, we calculated the degree of differential expression of miRNAs between normal oral mucosa and OSCC tissues. Twelve miRNAs (miR-31*, miR-31, miR135b, miR-193a-5p, miR-103, miR-224, miR-93, miR-200c, miR-183, miR-203, miR-21 and miR-223) were significantly upregulated in most of the cancer tissue samples compared with the normal oral mucosa. Those showing $>4$-fold upregulation were considered candidate miRNAs; namely, miR-31*, miR-31, miR-135b, miR-193a-5p, miR-103, miR-224, miR-93, miR-200c, miR-183, miR-203, miR-21 and miR-223. 
miR-21 has been investigated widely in various human malignancies including hematological malignancies and glioblastoma as a putative oncogenic miRNA $(5,19-26)$. Moreover, aberrantly expressed miR-203 has been detected in colon (27), breast (28) and ovarian cancer (29-31), while miR-31 is also known to have oncogenic functions in esophageal cancer (32). Upregulation of both miR-31 and miR-31* by delivery of pre-miR-31 was also shown to enhance OSCC oncogenicity (33). Our results also indicated that miR-31 and miR-31* have a biological function in cancer development. However, a role in oral cancer has yet to be documented, and the function of these candidates in human cancer remains unclear. miR-133a, miR-376c, miR-411, miR-30a-3p, miR-489, miR-139-5p, miR-483-5p, miR-30e-3p, miR-409-3p, let-7c and miR-486-5p showed a $>4$-fold downregulation (Table III). Previously, miR-133a was shown to be downregulated in breast cancer tissues and correlated with poor prognosis (34), while let-7c is reportedly related to tumor growth inhibition in prostate cancer (35).

Cervical lymph node metastasis has a large impact on the prognosis of OSCC. We, therefore, performed volcano plot analysis according to the metastatic state. Three downregulated miRNAs (niR-489, miR-1291 and miR-483-5p) (Table IV) were detected, and subsequently, a decision tree model was constructed to classify non-metastatic and metastatic OSCC using Weka software. Our model divided the two groups with $82 \%$ accuracy (Fig. 3). This model may therefore have potential in determining the prognosis of OSCC patients, acting as a predictive marker of metastasis.

In summary, our findings identifying cancer-related miRNAs in OSCC suggest that oral cancer may have a unique miRNA expression pattern at the individual level. Further investigations are now required to determine the molecular functions and mechanisms of these miRNAs as well as their potential use as prognostic and/or diagnostic markers in oral cancer.

\section{Acknowledgements}

We thank Tomohide Isobe and Tetsuhiko Tachikawa for the diagnostic clinical oral cancer tissues.

\section{References}

1. Bernier J, Bentzen SM and Vermorken JB: Molecular therapy in head and neck oncology. Nat Rev Clin Oncol 6: 266-277, 2009.

2. Bozec A, Peyrade F, Fischel JL and Milano G: Emerging molecular targeted therapies in the treatment of head and neck cancer. Expert Opin Emerg Drugs 14: 299-310, 2009.

3. Hummel R, Maurer J and Haier J: MicroRNA: biogenesis, function and role in rancer. Curr Genomics 11: 537-561, 2010.

4. Wiemer EA: The role of microRNAs in cancer: no small matter. Eur J Cancer 43: 1529-1544, 2007.

5. Lu J, Getz G, Miska EA, et al: MicroRNA expression profiles classify human cancers. Nature 435: 834-838, 2005.

6. Calin GA and Croce CM: MicroRNA signatures in human cancers. Nat Rev Cancer 6: 857-866, 2006

7. Wu BH, Xiong XP, Jia J and Zhang WF: MicroRNAs: new actors in the oral cancer scene. Oral Oncol 47: 314-319, 2011.

8. Reis PP, Tomenson M, Cervigne NK, Machado J, Jurisica I and Pintilie M: Programmed cell death 4 loss increases tumor cell invasion and is regulated by miR-21 in oral squamous cell carcinoma. Mol Cancer 10: 238, 2010.

9. Gottardo F, Liu CG, Ferracin M, et al: Micro-RNA profiling in kidney and bladder cancers. Urol Oncol 25: 387-392, 2007.
10. Volinia S, Calin GA, Liu CG, et al: A microRNA expression signature of human solid tumors defines cancer gene targets. Proc Natl Acad Sci USA 103: 2257-2261, 2006.

11. Hammond SM: MicroRNA therapeutics: a new niche for antisense nucleic acids. Trends Mol Med 12: 99-101, 2006.

12. Lagos-Quintana M, Rauhut R, Lendeckel W and Tuschl T: Identification of novel genes coding for small expressed RNAs. Science 294: 853-858, 2001.

13. Bartel DP: MicroRNAs: genomics, biogenesis, mechanism, and function. Cell 116: 281-297, 2004.

14. Ruan K, Fang X and Ouyang G: MicroRNAs: novel regulators in the hallmarks of human cancer. Cancer Lett 285: 116-126, 2009.

15. Farazi TA, Spitzer JI, Morozov P and Tuschl T: miRNAs in human cancer. J Pathol 223: 102-115, 2011.

16. Calin GA, Liu CG, Sevignani C, et al: MicroRNA profiling reveals distinct signatures in B cell chronic lymphocytic leukemias. Proc Natl Acad Sci USA 101: 11755-11760, 2004.

17. Schaefer A, Jung M, Mollenkopf HJ, et al: Diagnostic and prognostic implications of microRNA profiling in prostate carcinoma. Int J Cancer 126: 1166-1176, 2010.

18. Schepeler T, Reinert JT, Ostenfeld MS, et al: Diagnostic and prognostic microRNAs in stage II colon cancer. Clin Chem 54: 1696-1704, 2008

19. Zhang B, Pan X, Cobb GP and Anderson TA: microRNAs as oncogenes and tumor suppressors. Dev Biol 302: 1-12, 2007.

20. Papagiannakopoulos T, Shapiro A and Kosik KS: MicroRNA-21 targets a network of key tumor-suppressive pathways in glioblastoma cells. Cancer Res 68: 8164-8172, 2008.

21. Rossi L, Bonmassar E and Faraoni I: Modification of miR gene expression pattern in human colon cancer cells following exposure to 5-fluorouracil in vitro. Pharmacol Res 56: 248-253, 2007.

22. Cheng Y, Ji R, Yue J, et al: MicroRNAs are aberrantly expressed in hypertrophic heart: do they play a role in cardiac hypertrophy? Am J Pathol 170: 1831-1840, 2007.

23. Liu X, Cheng Y, Zhang S, Lin Y, Yang J and Zhang C: A necessary role of miR-221 and miR-222 in vascular smooth muscle cell proliferation and neointimal hyperplasia. Circ Res 104: 476-487, 2009.

24. Zhu S, Wu H, Wu F, Nie D, Sheng S and Mo YY: MicroRNA-21 targets tumor suppressor genes in invasion and metastasis. Cell Res 18: 350-359, 2008

25. Meng F, Henson R, Wehbe-Janek H, Ghoshal K, Jacob ST and Patel T: MicroRNA-21 regulates expression of the PTEN tumor suppressor gene in human hepatocellular cancer. Gastroenterology 133: 647-658, 2007.

26. Löffler D, Brocke-Heidrich K, Pfeifer G, et al: Interleukin-6 dependent survival of multiple myeloma cells involves the Stat3mediated induction of microRNA-21 through a highly conserved enhancer. Blood 15: 1330-1333, 2007.

27. Schetter AJ, Leung SY, Sohn JJ, Zanetti KA, Bowman ED and Yanaihara N: MicroRNA expression profiles associated with prognosis and therapeutic outcome in colon adenocarcinoma. JAMA 299: 425-436, 2008.

28. Iorio MV, Ferracin M, Liu CG, et al: MicroRNA gene expression deregulation in human breast cancer. Cancer Res 65: 7065-7070, 2005.

29. Nam EJ, Yoon H, Kim SW, Kim H, Kim YT and Kim JH: MicroRNA expression profiles in serous ovarian carcinoma. Clin Cancer Res 14: 2690-2695, 2008.

30. Saini S, Majid S, Yamamura S, et al: Regulatory role of mir-203 in prostate cancer progression and metastasis. Clin Cancer Res 17: 5287-5298, 2011

31. Wellner U, Schubert J, Burk UC, et al: The EMT-activator ZEB1 promotes tumorigenicity by repressing stemness-inhibiting microRNAs. Nat Cell Biol 11: 1487-1495, 2009.

32. Alder H, Taccioli C, Chen H, et al: Dysregulation of miR-31 and miR-21 induced by zinc deficiency promotes esophageal cancer. Carcinogenesis 33: 1736-1744, 2012.

33. Chang KW, Kao SY, Wu YH, et al: Passenger strand miRNA miR-31(*) regulates the phenotypes of oral cancer cells by targeting RhoA. Oral Oncol 49: 27-33, 2013.

34. Wu ZS, Wang CQ, Xiang R, et al: Loss of miR-133a expression associated with poor survival of breast cancer and restoration of miR-133a expression inhibited breast cancer cell growth and invasion. BMC Cancer 12: 51, 2012.

35. Sugimura K, Miyata $\mathrm{H}$, Tanaka K, et al: Let-7 expression is a significant determinant of response to chemotherapy through the regulation of IL-6/STAT3 pathway in esophageal squamous cell carcinoma. Clin Cancer Res 18: 5144-5153, 2012. 